\title{
An estimation of the UV radiation inside the cockpits of large commercial jets
}

\author{
Ralf Meerkötter ${ }^{1}$
}

Received: 31 July 2015/Revised: 27 October 2016/ Accepted: 7 November 2016

(C) Deutsches Zentrum für Luft- und Raumfahrt e.V. 2016

\begin{abstract}
UV irradiances and UV doses inside the cockpit of large commercial jets are estimated. Results are based on radiative transfer calculations taking into account the spectral transmittances and the limited fields of view of large commercial jet windscreens. In a first step, vertical profiles of UV irradiances for a cloud free atmosphere over snow free and snow covered surfaces and for an atmosphere containing a water cloud layer are simulated. It turns out that the windscreens block the UV-B radiation and transmit parts of the UV-A radiation. Comparing UV irradiances inside and outside the cockpit show that the intensity of UV radiation inside strongly depends on whether the direct sun is entering the cabin or not. Without direct sun the diffuse UV radiation inside the cockpit amounts to about $5 \%$ the ambient UV irradiance outside the aircraft. In cases of low sun when direct radiation can reach the pilot, percentages grow from 50 to $100 \%$ with increasing solar zenith angle. A water cloud layer between 2 and $4 \mathrm{~km}$ increases the UV irradiances inside a cockpit by about $7 \%$ at $10 \mathrm{~km}$ altitude when compared to the cloud free atmosphere. A snow covered surface causes a similar increase. Finally, and by the aid of MOZAIC waypoint data UV doses were estimated for selected long-distance flights between Europe and the overseas continents North and South America, South Africa, and East Asia. UV doses are affected by takeoff and landing time, by the sun position relative to the aircraft heading during flight, and by the day of the year. UV doses inside the cockpit amount to maximum $60 \%$ the UV doses outside at the same altitude,
\end{abstract}

Ralf Meerkötter

ralf.meerkoetter@dlr.de

1 Institut für Physik der Atmosphäre, Deutsches Zentrum für Luft- und Raumfahrt (DLR), Oberpfaffenhofen, Germany however, in most cases percentages are between about 10 and $40 \%$.

Keywords UV radiation - UV irradiance - UV dose . Cockpit · Pilots - Commercial jets - Radiative transfer simulations

$\begin{array}{ll}\text { Abbreviations } \\ \text { ATL } & \text { Atlanta, IATA-code } \\ \text { AOT } & \text { Aerosol optical thickness } \\ \text { BRU } & \text { Brussels, IATA-code } \\ \text { COT } & \text { Cloud optical thickness } \\ \text { DU } & \text { Dobson units } \\ \text { FRA } & \text { Frankfurt, IATA-code } \\ \text { GRU } & \text { Sao Paulo, IATA-code } \\ \text { IATA } & \text { International Air Transport Association } \\ \text { MOZAIC } & \text { Measurement of ozone and water vapor on } \\ & \text { airbus in-service aircraft } \\ \text { NRT } & \text { Tokyo, IATA-code } \\ \text { SZa } & \text { Solar zenith angle } \\ \text { UV } & \text { Ultraviolet } \\ \text { UV-A } & \text { Ultraviolet-A } \\ \text { UV-B } & \text { Ultraviolet-B } \\ \text { WDH } & \text { Windhoek, IATA-code }\end{array}$

\section{List of symbols}

$\Delta \delta \quad$ Viewing zenith angle interval $\left(^{\circ}\right)$

$\Delta \varphi \quad$ Viewing azimuth angle interval $\left(^{\circ}\right)$

f $\pi / 180$

$L_{\lambda} \quad$ Spectral irradiance $\left(\mathrm{Wm}^{-2} \mathrm{~nm}^{-1}\right)$

$\lambda \quad$ Wavelength (nm)

$r \quad$ Ratio of wavelength integrated irradiances

$T_{\lambda} \quad$ Spectral windscreen transmittance $\lambda$ Wavelength (nm)

$\omega \quad$ Solid angle (sr) 


\section{Introduction}

Solar ultraviolet radiation (UV) is defined in the wavelength interval from 100 to $400 \mathrm{~nm}$ which is further divided into the sub-intervals of UV-C (100-280 nm), UV-B (280-315 nm), and UV-A (315-400 nm). Whereas UV-A and parts of the UV-B reach the earth's surface, UV-C is almost completely filtered in the upper layers of the atmosphere. The absorption of UV-C and UV-B radiation is mainly caused by the stratospheric ozone layer between about 12 and $50 \mathrm{~km}$ altitude. At the short UV wavelengths photons carry higher amounts of energy compared to longer wavelengths, as for example the wavelengths of visible light. As a result, UV radiation is potentially more damaging to tissues of humans, animals, and plants than radiation at wavelengths greater $400 \mathrm{~nm}$.

The risk of overexposure to UV radiation is well known and associated various adverse effects on the human health have been described in numerous studies and reports (e.g., [1] and related studies, [2]). Human behavior and particular occupational conditions can lead to detrimental UV doses if adequate preventive measures cannot or are not be taken or if they were ineffective.

This study addresses the UV radiation intensity inside airliner cockpits. Whether airliner pilots are exposed to critical levels of UV radiation has to-date been addressed only in a few studies. Diffey and Roscoe [3] measured UV radiation exposure during 12 flights using a polysulphone film badge worn by pilots. The sensitivity of the film was confined to wavelengths less than $320 \mathrm{~nm}$. All badges worn during flight show minimal exposure to UV radiation and were significantly less than the radiation outside at ground level. However, details regarding measurements procedures, accuracies of the film measurements, or the spectral sensitivities of the films were not given. The review paper of Chorley et al. [4] summarizes that studies measuring cockpit UV radiation are limited and leave unanswered questions regarding airline pilot exposure and that although aircraft windshields block UV-B radiation the intensity of UV-A within the cockpit at altitude is unknown. On the other hand, there are indications that UV-A radiation has mutagenic and carcinogenic effects on human skin (e.g., $[5,6]$ ) and potentially damaging effects on the eye (e.g., [7]). Sanlorenzo et al. [8] conclude that cumulative UV exposure for pilots and cabin crew is still of concern, but they also state that it is not clear whether health risks are causally associated with exposures during flight. Based on UV measurements in a flying general aviation turboprop airplane Sanlorenzo et al. [9] found that windshields block UV-B radiation but allowed UV-A transmission. The authors further state that at $9000 \mathrm{~m}$, where the most aircraft fly, the UV level is approximately twice that of the ground and that these levels can be even higher over thick cloud layers or snow fields which could reflect up to $85 \%$ of UV radiation $[8,9]$. They recommend further studies and UV measurements in several airplanes. Chorley et al. [10] describe the development of instrumentation for automated time-stamped spectral measurements and tested the equipment already in a number of aircraft and helicopter flights during 2012-2013. The instrument would enable to ascertain variances and maxima of UV exposures during flight.

This paper takes up the question about UV-A radiation levels inside a cockpit conditioned by windscreen properties, by flight route parameters, and by the atmosphere. It focuses on quantifying the share of UV radiation that is potentially reaching a pilot inside the cockpit in relation to ambient UV radiation levels outside the aircraft. Physiological effects of UV radiation penetrating the human skin or the eye are not subject of this study and associated health risks due to UV exposure, as for example damages of the eye (e.g., cataracts) or the human skin (e.g., melanoma), are not examined and discussed. Likewise the protection by sunglasses, certain types of spectacles, or contact lenses is not studied.

To obtain meaningful data by manageable effort an approach based on radiative transfer calculations has been chosen. Radiative transfer simulations allow for a comparison of UV irradiances and UV doses inside and outside the aircraft cockpit under various conditions. This regards for example relevant atmospheric parameters, aircraft heading in relation to the position of the sun, and aircraft windscreen design parameters. At first, it is shown how UV irradiances behave as a function of altitude in a cloud free atmosphere, in a cloudy atmosphere, and over a snow covered ground. Then, by the use of Measurement of Ozone and Water vapor on Airbus in-service Aircraft (MOZAIC) waypoint data, cumulative UV doses are calculated for real flights that have been operated on routes from Europe to airports in different overseas continents as North and South America, South Africa, and Eastern Asia at different times and dates of the year. From the MOZAIC data, only the waypoint information has been extracted which gives the relative solar position during flight. Clouds and a varying surface reflection due to varying snow or ice have not been taken into account for the calculation of UV doses based on MOZAIC data. Finally, results are analyzed and discussed.

\section{Approach}

Outside an aircraft, UV radiation primarily depends on solar zenith angle, altitude, total ozone column amount, UV surface albedo, and on the three dimensional 
distribution of clouds. Inside an aircraft, cockpit UV radiation is spectrally filtered according to the specific transmission characteristics of an aircraft windscreen. The UV intensity particularly depends on whether radiation of the direct sun is entering the cockpit and reaching the pilot during flight or not. Therefore, the main parameters determining the UV intensity inside a cockpit are the actual solar zenith and azimuth angle in relation to the aircraft heading, the windscreen spectral transmittance, and the windscreen geometry that is spanning a specific solid angle relative to the position of the target of interest. The approach chosen to quantify UV irradiances inside and outside an aircraft cockpit is based on radiative transfer model simulations.

\subsection{Radiative transfer model}

The radiative transfer model employed is a one-dimensional model based on the doubling-adding-method which is known as one of the most accurate tools for describing the relevant processes of multiple scattering and absorption (e.g., [11]). A model version for the UV spectral range has been developed and successfully been utilized in many applications, as for example for the calculation of surface UV based on satellite measurements (e.g., [12, 13]).

The model generates spectral irradiances in a wavelength resolution of $1 \mathrm{~nm}$ over the spectral range from 290 to $400 \mathrm{~nm}$. Output are vertical profiles of spectral irradiances at each boundary of the atmospheric layers from the ground to an altitude of $100 \mathrm{~km}$. Up to an altitude of $12 \mathrm{~km}$ the vertical resolution is $1 \mathrm{~km}$, above $12 \mathrm{~km}$ it is $2 \mathrm{~km}$, and above $32 \mathrm{~km}$ the resolution is $5 \mathrm{~km}$ and less. Incident solar radiation is discretized into 12 solar zenith angles (sza) including the angle of $0^{\circ}$. With respect to the use of the MOZAIC waypoint data (date, time, latitude, longitude, and altitude) calculated irradiances are used to generate a fit-algorithm which allows for the interpolation of the discretized model output. Wavelength integration from 290 to $400 \mathrm{~nm}$ is performed without convolution of any biological action spectrum, but for UV irradiances inside the cockpit spectral windscreen transmittances are taken into account.

Temporal integration of spectrally integrated UV irradiances results in UV doses. For the calculation of UV doses from takeoff to landing of an airplane, the program code allows to work with synthetic as well as with real waypoint data. MOZAIC waypoint data are recorded with a temporal resolution of $4 \mathrm{~s}$, however, for the integration of UV doses the resolution has been reduced to time steps of about 2 min. Based on date, time, latitude, longitude, and aircraft heading the code determines whether the sun disk is located inside or outside a windscreen's field of view. Depending on situation it either calculates the irradiance as the sum of direct and diffuse radiation or as the contribution from diffuse radiation only.

\subsection{Model atmosphere}

For describing the effects of Rayleigh scattering and ozone absorption the vertical profiles of temperature, air density, and ozone density have been taken from the midlatitude summer standard atmosphere [14]. The total ozone column amount, however, is fixed to $320 \mathrm{DU}$. This assumption is justified, since as shown below, UV radiation behind the considered aircraft windscreens is reduced to the UV-A part $(315-400 \mathrm{~nm})$ where ozone absorption is negligible. For a snow-free surface, a UV albedo of 0.03 is assumed, whereas a snow covered ground is represented by a value of 0.85 . An albedo of 0.85 is valid for a highly reflecting snow surface, and therefore appropriate to simulate maximum effects on UV. Albedo values of snow surfaces are usually depending on age, grain size, purity, structure or on the degree of mixing with other components [15]. By trend a real snow UV albedo will be lower than 0.85 but would imply smaller effects on UV. To simulate the radiation fields above clouds, a water cloud layer ranging from 2 to $4 \mathrm{~km}$ altitude is embedded in the model atmosphere. Spectral optical parameters as single scattering albedo, asymmetry parameter and extinction coefficient stem from separate Mie-calculations on the basis of a given particle size distribution and spectral complex refractive indices. Assumed is a cloud droplet concentration of $100 \mathrm{~cm}^{-3}$ which results in a cloud optical thickness (COT) of 45 at the wavelength $330 \mathrm{~nm}$. The atmospheric aerosol is described by a continental type. As it is the total aerosol optical thickness (AOT) which is mainly determining the UV intensity other aerosol types are not considered. Although the AOT is highly variable in space and time (e.g., [16]), it has negligible effects on UV radiation inside a cockpit at flight level. For this reason and due to the fact that mainly the radiation of longer wavelengths within the UV-A spectral range is transmitted into the cockpit $\mathrm{AOT}=0.2$ is assumed.

\subsection{Windscreen spectral transmittance}

Nakagawara et al. [7] performed transmittance measurements on several aircraft windscreens from large commercial jets, from a small private jet, and from commercial propeller-driven passenger planes. These measurements are certainly not representative for the entire variety of currently existing spectral windscreen transmittances manufactured for different aircrafts, but it is assumed that they are at least describing typical spectral transmission characteristics being adequate for simulations of UV irradiances and UV doses inside a cockpit. For the purpose of 
this study, the transmittance data obtained for the multilayer (laminated) composite glasses of the large commercial jets Airbus A320, Boeing 727/737, and MD 88 are selected. The data were digitized from the figures in [7], then fitted and interpolated to the spectral resolution of $1 \mathrm{~nm}$. UV transmittances for the glass windscreens of the Airbus A320 and the Boeing 727/737 are less than $1 \%$ over the entire UV-B spectral interval. For wavelengths greater than $315 \mathrm{~nm}$, i.e., within the UV-A, spectral transmittances increase with wavelength reaching values of about 65-70\% near $400 \mathrm{~nm}$. In comparison to the Airbus and Boeing curves, the MD88 transmittance is further reduced over a large domain within the UV-A. Nevertheless, multilayer glass windscreens block almost completely the more harmful UV-B radiation, but still allow for the transmission of UV-A radiation. This is illustrated in Fig. 1 displaying curves of spectral UV irradiances at the ground with and without any spectral windscreen filter. As can be seen, filtered irradiances will no longer depend on total ozone column amount which is effective in the UV-B (280$315 \mathrm{~nm}$ ) and wavelength integrated UV irradiances merely result from UV-A $(315-400 \mathrm{~nm})$ contributions. The reduction of wavelength integrated irradiances due to windscreens can be expressed by the ratio

$r=\frac{\int_{290 \mathrm{~nm}}^{400 \mathrm{~nm}} L_{\lambda} \times T_{\lambda} \times \mathrm{d} \lambda}{\int_{290 \mathrm{~nm}}^{400 \mathrm{~nm}} L_{\lambda} \times \mathrm{d} \lambda}$,

with $L_{\lambda}$ the spectral irradiance and $T_{\lambda}$ the spectral windscreen transmittance. At $10 \mathrm{~km}(0 \mathrm{~km})$ and for sza $=60^{\circ}$ $r$ results in 0.32 (0.34), $0.36(0.38)$, and $0.21(0.23)$ when considering the Airbus, Boeing, and MD88 windscreens, respectively. The ratios are largely independent of the sza. For example, sza $=0^{\circ}$ gives the respective numbers 0.31 (0.32), $0.34(0.36)$, and 0.20 (0.22). Slightly different values of $\mathrm{r}$ at $10 \mathrm{~km}$ and at $0 \mathrm{~km}$ altitude are due to

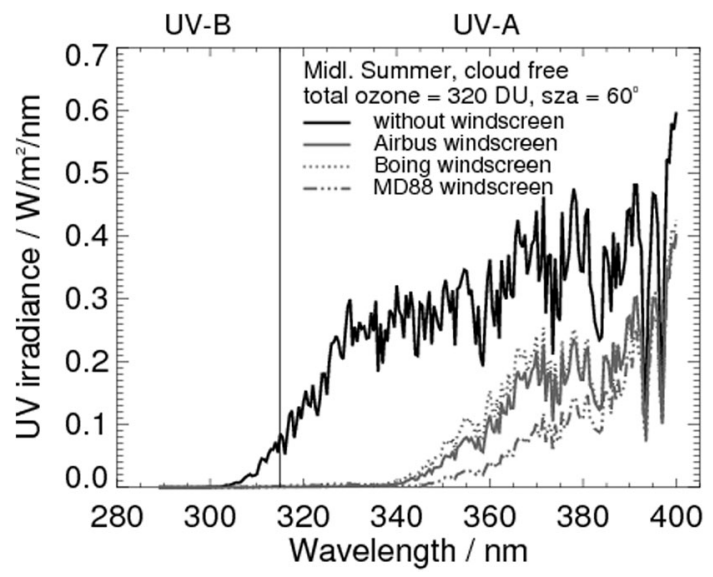

Fig. 1 Simulated spectral UV irradiances at a snow-free ground without and with filtering by different spectral windscreen transmittances of three commercial jets wavelength dependent extinction properties of aerosol particles and air molecules and by their respective vertical density profiles. Finally, it should be mentioned that Chorley et al. [4] point to possible changes of spectral windscreen transmission at altitude where different temperatures would be present. Nakagawara et al. [7] hint at multiple reflection effects within the breadboarding likely leading to measurements of higher transmittances for visible wavelengths. Due to the lack of corresponding data both these effects have not been taken into account.

\subsection{Windscreen geometry}

According to the specific windscreen geometry, UV radiation inside an aircraft cockpit is coming from an angular sector clearly smaller than that of the entire hemisphere with the consequence that a predominant part of ambient UV radiation is not reaching a pilot. In principal, this angular sector is a rather complex figure as it is spanned by the specific windscreen geometry and the distance from the windscreen to a point on the pilot's body being of interest. Under real conditions it is also varying as a function of time. To estimate the UV fraction that is transmitted into the cockpit, the overall dimensions of windscreens in height and horizontal extension have been approximated by a rectangular. Figure 2 exemplarily illustrates how the approximation fits to an Airbus A319/320/321 windscreen. Viewing angles indicated in Fig. 2 are valid for the assumption that the pilot's eyes define the distance to the target position for incident radiation, a configuration which has been taken for all simulations. Although design details are varying between different types of commercial jets, the viewing angle dimensions of different windscreens are quite similar (e.g., [17, 18]).

During flight only a small part of the pilot's skin is exposed to solar UV radiation, usually the face and the

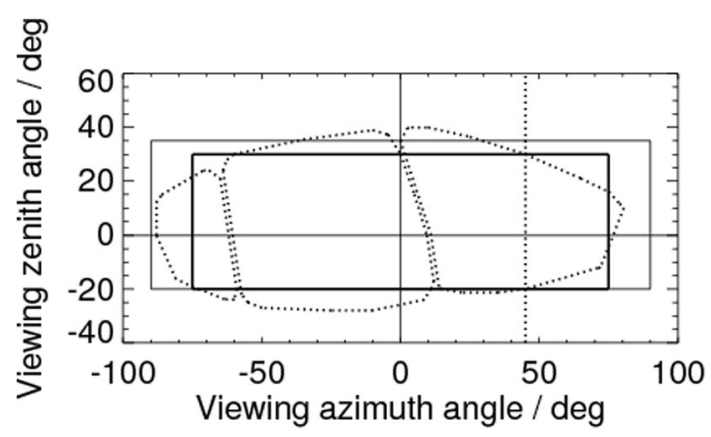

Fig. 2 Thick and thin solid lines rectangular windscreen approximations seen from a pilot's eye as used for UV simulations in this study. The vertical solid line represents the target axis, the viewing zenith angle refers to the normal on a vertically oriented plane, and the line at $0^{\circ}$ zenith angle marks the horizon. Dotted lines an Airbus A-320 windscreen with pilot axis (vertical line) as shown in [17] 
forearms. For assessing the potential UV exposure inside a cockpit maximum values are of interest which can primarily be expected for vertically oriented skin elements at the position of the pilot's head. Moreover, windscreen geometries pre-select directions of incoming radiation which provide maximum irradiance contributions for vertical elements (see results for direct sun below). Thus, simulations of the UV radiation inside the cockpit are performed with respect to a vertically oriented target area.

The solid angle $\omega$ of a rectangular windscreen can easily be obtained from:

$\omega=\arcsin (\sin (\Delta \delta \times f / 2) \times \sin (\Delta \varphi \times f / 2))$,

with $f=\pi / 180$ and $\Delta \delta$ and $\Delta \varphi$ denoting the intervals of viewing zenith and viewing azimuth angle, respectively. UV calculations are performed for $\Delta \delta=50^{\circ}$ and $\Delta \varphi=150^{\circ}$ with the target located at the bisecting line of the azimuth angle interval (Fig. 2). For the assessment of uncertainties $\Delta \delta=55^{\circ}$ and $\Delta \varphi=180^{\circ}$ have been used. The azimuth angle between incident direct solar radiation and the normal of the target area is assumed to be $0^{\circ}$ which implies that the target area is following the solar position. This, on the one hand, eases computation, but may also be justified because body facets are usually in motion around the vertical axis during flight. The vertical orientation of the target area is assumed to be fixed.

Modifications of UV caused by penetrating the windscreens along a slant path are neglected. As shown in Fig. 2 the field of view ranges to $20^{\circ}$ below the horizon which implies that a fraction of upward directed UV radiation originating from scattering on air molecules, aerosol particles or from the reflection of cloud tops below the aircraft can reach the pilot. In a cloud free atmosphere reflected radiation especially stems from snow or ice covered land and ocean surfaces. The share of upward directed UV radiation is taken into account in all radiative transfer simulations.

\section{Results}

In the following UV irradiances and doses inside and outside an airliner cockpit are compared. Irradiances of atmospheric UV radiation are usually defined for a horizontally oriented plane and their order of magnitude is well known from diverse measurements or computations. Therefore, the irradiances and doses representing ambient UV conditions outside the airplane and used here as a reference, are referred to the horizontal plane. To estimate the upper limit of the share of ambient UV radiation which after penetrating a windscreen potentially reaches the pilot's skin, UV irradiances inside a cockpit are calculated for a vertically oriented plane. Note that for $\mathrm{sza}=60^{\circ}$ $\left(76^{\circ}\right)$ irradiances referred to a horizontal plane amount to about $60 \%(25 \%)$ the irradiances referred to a vertical plane. For the calculation of vertical profiles of UV irradiances (next subchapter) the azimuth angle between the aircraft roll axis and the direction to the sun is assumed to be $0^{\circ}$. During real flights (chapter after the next) the azimuth angle changes with aircraft heading, aircraft position and time. As far as it concerns, the position of the sun in relation to the windscreen's field of view this is considered, but for calculating UV doses it is assumed that the vertically oriented target is following the azimuth direction to the sun.

\subsection{Vertical profiles of $\mathrm{UV}$ irradiances}

Of particular interest is the question how much of the ambient UV radiation outside the cockpit reaches the interior of the cockpit. Figure 3 shows vertical profiles of calculated UV irradiances inside and outside an aircraft cockpit for a cloud free atmosphere (Fig. 3a), for an atmosphere containing a water cloud (Fig. 3b), and for an atmosphere with an underlying snow covered ground (Fig. 3e). Figure 3c, d display profiles of ratios relating UV irradiances inside the cockpit to those outside. $\mathrm{Sza}=66^{\circ}$ and $\mathrm{sza}=76^{\circ}$ represent situations with direct and diffuse solar UV radiation entering the cockpit. For sza $=40^{\circ}$ the sun is located above the windscreen's field of view with the consequence that only diffuse UV radiation is effective.

Irradiances of UV radiation outside an aircraft decrease with an increasing sza due to the extinction along longer atmospheric path lengths. Figure $3 a$, b show that over the entire altitude range absolute irradiance values for sza $=66^{\circ}$ are a factor of two smaller than those for sza $=40^{\circ}$. For sza $=76^{\circ}$ (not shown) it is a factor of four.

The ratio of irradiances inside and outside the airplane is 0.05 at all altitudes for sza $=40^{\circ}$ when only diffuse radiation reaches the cockpit (Fig. 3c). At a lower sun, when incident radiation includes the direct component $\left(\mathrm{sza}=66^{\circ}\right)$, ratios vary from 0.45 at $0 \mathrm{~km}$ to 0.65 at $10 \mathrm{~km}$. For sza $=76^{\circ}$ corresponding ratios lie in the range from 0.45 to 1.0 , but at this sza absolute irradiances are significantly reduced. Note that contributions of the direct sun to a hemispheric irradiance on a horizontally oriented plane are weighted with the cosine of large szas, whereas for the vertically oriented target area inside the cabin contributions are weighted with the cosine of small szas.

Compared to the ground the UV intensity at $10 \mathrm{~km}$ altitude is higher due to a less extinction of incoming radiation by the atmosphere. In case of the cloud free atmosphere and outside the cockpit UV irradiances at $10 \mathrm{~km}$ altitude are 30,50, and $70 \%$ higher for $\mathrm{sza}=40^{\circ}$, sza $=66^{\circ}$, and sza $=76^{\circ}$ (not shown) than those at the 
Fig. 3 Vertical profiles of simulated UV irradiances inside and outside the cockpit and their respective ratios for different solar zenith angles in a cloud free atmosphere (a, c), a cloudy atmosphere (b, d), and an atmosphere with an underlying snow surface (e)
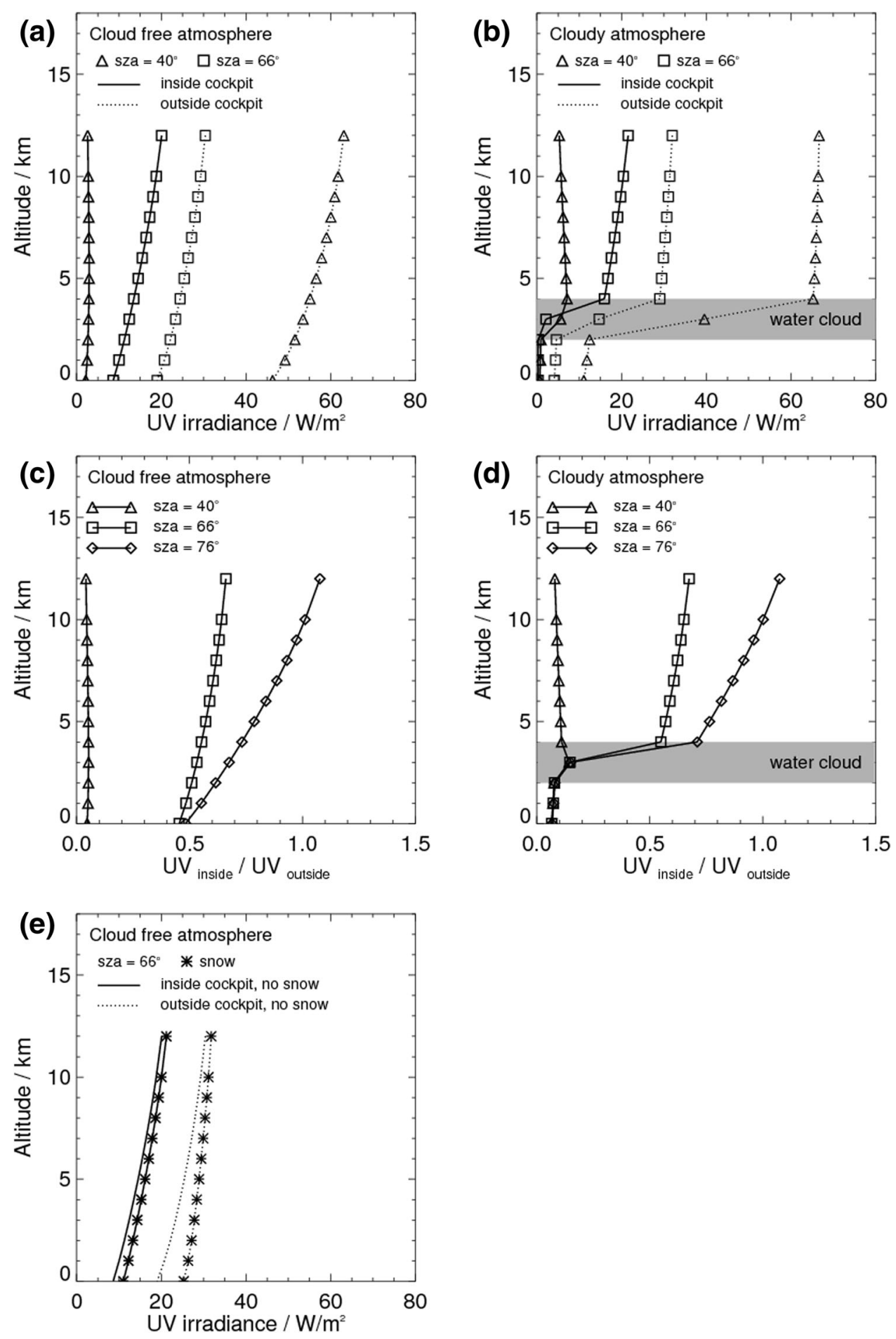

ground (Fig. 3a). Inside the cockpit the respective values are 30,120 , and $256 \%$. Percentages above $100 \%$ are occurring for conditions with direct radiation. The relative increase of direct radiation with altitude is larger because the effective optical thickness of the atmosphere decreases more strongly along a slant path. However, relative increases are high for low absolute irradiances.
Below the water cloud layer and outside the cockpit, UV irradiances are significantly reduced in comparison to values above the cloud (Fig. 3b). UV irradiances at the ground are, for example, 6.0, 7.6, and 8.3 times smaller than irradiances at $10 \mathrm{~km}$ for sza $=40^{\circ}$, sza $=66^{\circ}$, and sza $=76^{\circ}$ (not shown), respectively. Reflection processes at the cloud top enhance the UV radiation at all layers 
Fig. 4 Intercontinental flight routes selected for the calculation of UV doses based MOZAIC waypoint data. Starting point in Europe is Frankfurt (FRA), for the flight to Tokyo (NRT) it is Brussels (BRU). Although optically not resolvable, each route comprises eight flights (four dates, outbound and inbound)

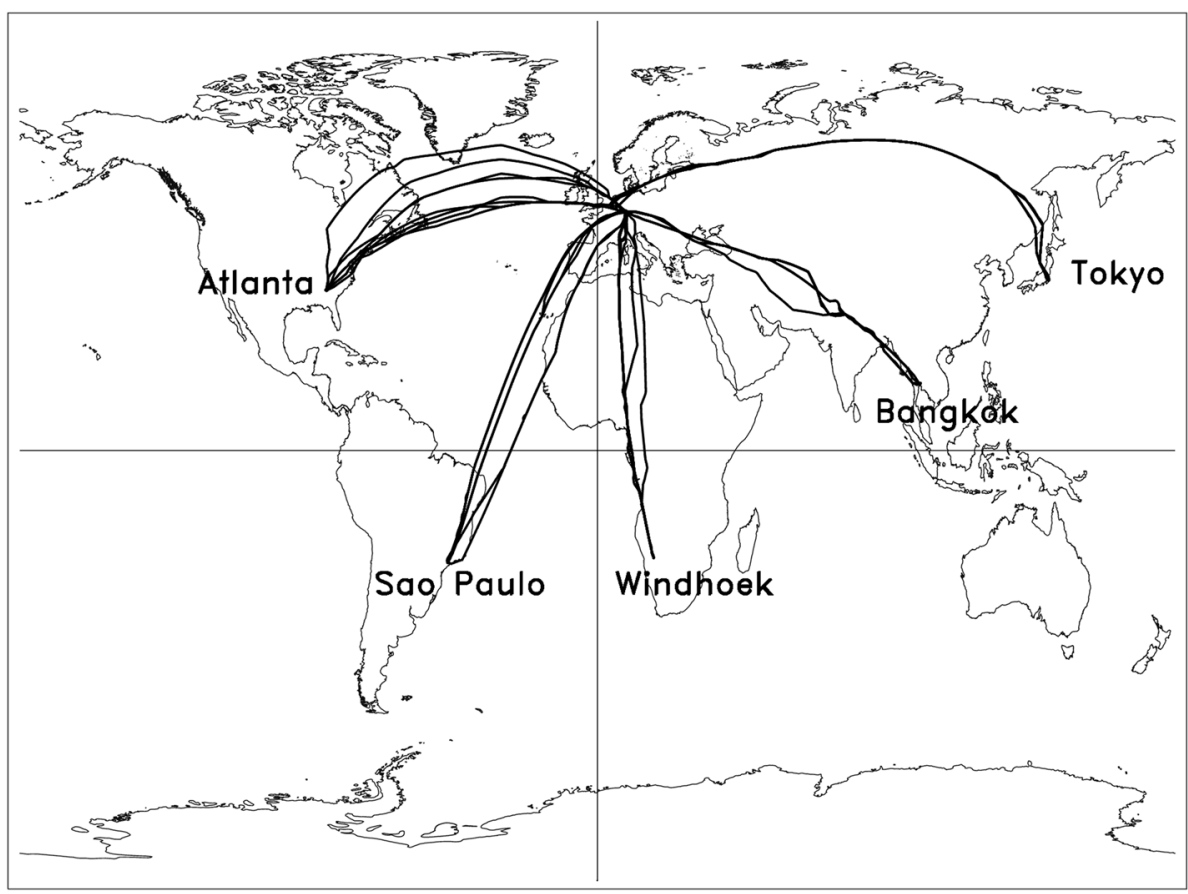

above the cloud. For example, at $10 \mathrm{~km}$ UV irradiances are about $7.0 \%$ higher than in the cloud free atmosphere for sza $=40^{\circ}$ and sza $=66^{\circ}$ (Fig. 3a, b). Directly at cloud top the enhancement even amounts to about $19 \%$. Reason is the higher air density at these altitudes amplifying multiple scattering processes between the cloud and atmospheric layers above. Above the cloud and in cases with direct sun $\left(\mathrm{sza}=66^{\circ}, 76^{\circ}\right)$ irradiance ratios are almost the same for the cloud free and the cloudy atmosphere (Fig. 3c, d). Within and below the cloud layer diffuse radiation is dominating leading to ratios similar to those for sza $=40^{\circ}$ (Fig. 3d). Note, the windscreen geometry allows that parts of the cloud layer below the horizon are within the field of view (Fig. 2).

Finally, Fig. 3e displays irradiance profiles for a cloud free atmosphere over a snow free and a snow covered ground. Irradiance differences are noticeable in the lower kilometers of the troposphere where multiple scattering processes are more effective. At the ground and outside the cockpit a snow cover increases UV irradiances by about $33 \%$, whereas at $10 \mathrm{~km}$ altitude the increase is reduced to about $6 \%$. Inside the cockpit the respective enhancements are 28 and $7 \%$. UV irradiance ratios (not shown) are almost the same as for the case without snow cover. It should be noted that at $10 \mathrm{~km}$ altitude UV increases due to snow are comparable to that caused by a cloud layer (Fig. 3a, b).

\subsection{UV doses during flight}

Of particular interest is the cumulative UV dose pilots may potentially be exposed to during flight. However, it is not the purpose of this study to provide total individual UV doses pilots receive during their flights over a year or during their entire occupational life. Calculations are rather made to obtain estimations of UV doses being typical for selected overseas long-range flights and to understand what is determining their order of magnitude.

UV doses depend on flight route, integrated flight time, sun position during flight from takeoff to landing, and the day of the year. Dose values presented in the following are based on the assumption that the aircraft is equipped with a windscreen having an Airbus spectral transmittance and a design corresponding to the approximated rectangular shape as shown in Fig. 2 (thick solid line) and described above. For the calculation of UV doses waypoint data recorded during the ascent, descent, and cruise phases of real intercontinental flights have been used as input. Geolocalization data stem from the MOZAIC (Measurement of Ozone and Water vapor on Airbus in-service Aircraft) database, an outcome of a research project funded by the EC between 1993 and 2004. Criterion for data selection is a takeoff in Europe with outbound and inbound directions representing routes to the West, to South-West, to the South, to South-East, and to the East (Fig. 4). Since the position of the sun and length of the day is varying over the year, UV doses are first calculated for flight routes on days around the solstices and equinoxes (Table 1, symbols in Fig. 5). Under the assumption that flight routes on these days are valid during the entire summer or winter schedules (changes on last sundays in March and October), UV doses are also calculated as a function of the day of the year (lines in Fig. 5). 
Table 1 MOZAIC flights operated around the solstices and equinoxes which have been selected for simulations

\begin{tabular}{|c|c|c|c|c|c|c|c|}
\hline \multirow[t]{2}{*}{ Route } & \multicolumn{4}{|l|}{ Date } & \multicolumn{3}{|l|}{ Time/UTC } \\
\hline & March & June & September & December & Takeoff & Landing & Flying \\
\hline FRA-ATL & 19.03 .00 & 20.06 .99 & 20.09 .00 & 22.12.99 & 08:57/09:59 & 18:41/20:01 & 09:44/10:02 \\
\hline ATL-FRA & 19.03 .00 & 20.06 .99 & 20.09 .00 & 22.12.99 & $21: 01 / 22: 12$ & 04:59/05:30 & 07:58/07:18 \\
\hline FRA-GRU & 21.03 .99 & 18.06.99 & 19.09 .99 & 20.12.99 & $20: 16 / 21: 21$ & 07:49/09:39 & $11: 33 / 12: 18$ \\
\hline GRU-FRA & 22.03 .99 & 19.06.99 & 20.09 .99 & 21.12.99 & $16: 51 / 17: 36$ & 04:00/04:39 & 11:09/11:03 \\
\hline FRA-WDH & 19.03 .99 & 20.06 .98 & 24.09 .98 & 20.12.99 & 08:46/21:58 & $18: 22 / 07: 21$ & 09:36/09:23 \\
\hline WDH-FRA & 19.03 .99 & 20.06 .98 & 24.09 .98 & 21.12.99 & 20:11/18:55 & 05:50/04:54 & 09:38/09:59 \\
\hline FRA-BKK & 20.03 .99 & 22.06.97 & 19.09 .97 & 24.12.97 & 16:08/16:59 & 02:55/03:36 & $10: 47 / 10: 37$ \\
\hline BKK-FRA & 21.03 .99 & 23.06.97 & 20.09 .97 & 25.12.97 & 05:17/05:21 & $16: 58 / 17: 42$ & $11: 41 / 12: 21$ \\
\hline BRU-NRT & 20.03 .98 & 24.06.98 & 23.09 .98 & 21.12.00 & $13: 33 / 14: 35$ & 01:01/02:22 & $11: 28 / 11: 47$ \\
\hline NRT-BRU & 30.03 .98 & 25.06.99 & 24.09 .98 & 22.12.00 & 02:59/04:12 & 14:39/16:06 & 11:40/11:54 \\
\hline
\end{tabular}

Data in the last three columns belong to boldfaced dates. On corresponding days in March and September takeoff, landing, and flying times are similar

Airports are abbreviated according to the IATA-code: ATL Atlanta, BKK Bangkok, BRU Brussels, FRA Frankfurt, GRU Sao Paulo, NRT Tokyo, WDH Windhoek
Figure 5a, b display UV doses calculated by temporal integration of UV irradiances from takeoff to landing for flights along the routes displayed in Fig. 4. Symbols mark UV doses obtained for days listed in Table 1, whereas lines result under the assumption that flight routes on days around the equinoxes and solstices are the same for every day during the summer or winter flight schedule. Striking are UV doses for flights on the route FRA-GRU being zero for the most time of the year except for days in December when UV doses increase to at most $9 \mathrm{kJm}^{-2}$ (Fig. 5a). Reason is that flights to GRU are predominantly operated during night. In December, only diffuse UV radiation is reaching the interior of the cockpit and this for about 90 min before landing. Direct UV radiation reaches the cockpit for only a few minutes at the end of the flight. A relatively strong increase of UV doses results for flights on the route FRA-ATL in the winter months. For example, on 22.12.1999 the aircraft is flying against a low sun for about $45 \%$ of the flight time which means that direct radiation contributes to the UV dose for the almost last $5 \mathrm{~h}$. UV doses for all other flights outbound Europe remain between 70 and $200 \mathrm{kJm}^{-2}$.

Highest UV doses between about 380 and $600 \mathrm{kJm}^{-2}$ are calculated for flights back from East Asia, i.e., on westwards routes in March and September (Fig. 5b). On days around the spring and autumn equinoxes in March and September, the corresponding solar zenith angles are greater than in summer. As a consequence, the direct sun is effective during the afternoon flight hours (local time) on these routes, especially when the airplanes are approaching Europe. For example, on the route BKK-FRA (NRT-BRU) direct sun contributes to the total UV dose by $46 \%$ (70\%) of the flight time on 21.03.1999 (30.03.1998), whereas on 23.06.1997 (25.06.1999) the percentage is reduced to $17 \%$
(0\%). Note, the flight NRT-BRU is operated at higher latitudes than the flight BRU-FRA (Fig. 4). On 25.12.1997 (22.12.1999) the direct sun is effective for $21 \%(0 \%)$ of the flight time on the route BKK-FRA (NRT-BRU).

Figure $5 \mathrm{c}$ and $\mathrm{d}$ relate UV doses inside the cockpit to those outside at flight altitude. Regarding the flights from FRA to Asia (Fig. 5c), UV doses inside the cockpit are about $20-30 \%$ the doses outside for days in March, June, and September. For the flights FRA-ATL and FRA-WDH percentages are even reduced to about $5 \%$ in the same months. In December, ratios reach about $60 \%$ because larger solar zenith angles increase the contribution of direct radiation to UV doses. For routes back to Europe (Fig. 5d), ratios are remaining below 50\%. Peak values about 85 and $65 \%$ result for the flight NRT-BRU in February and November, respectively, but these percentages are not coinciding with the maxima of absolute UV doses (Fig. 5b).

Figure 5e presents UV doses inside the cockpit in relation to UV doses outside at the ground and at the location of departure. UV doses at ground are calculated for the interval of flying time but with a halftime at local noon, thus potentially leading to maximum UV doses. The ratios reach one maximum of about $45 \%$ for the flight FRA-BKK in December and two maxima of almost $60 \%$ on the route NRT-BRU in March and September. These maxima are associated with flights on which the direct sun reaches the cockpit over longer time intervals. For the other flights, however, ratios are clearly below $10 \%$ which, for example, would mean that persons staying outdoors for the same time interval at ground receive an at least ten times higher UV dose.

From results presented in Fig. 5 it is obvious that UV doses inside a cockpit are clearly reduced (by about 
Fig. 5 a UV doses inside a cockpit from takeoff to landing for outbound flights from Europe. Symbols mark calculations for days listed in Table 1, lines represent day-today calculations assuming that flight routes around equinoxes and solstices are valid during the summer or winter schedules. b same as in a but for flights back to Europe. c UV doses inside the cockpit in relation to UV doses outside at flight altitude for outbound flights from Europe and for routes of high UV doses. d same as in c but for flights back to Europe. e UV doses inside the cockpit in relation to UV doses outside at the ground at the location of departure (a)

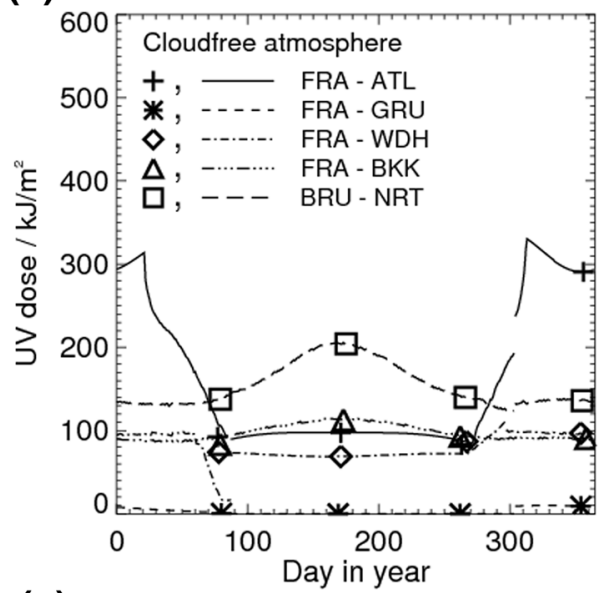

(c)

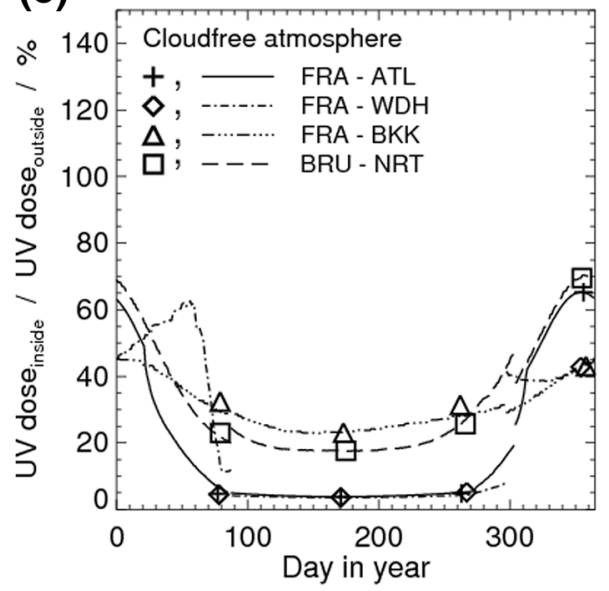

(e)

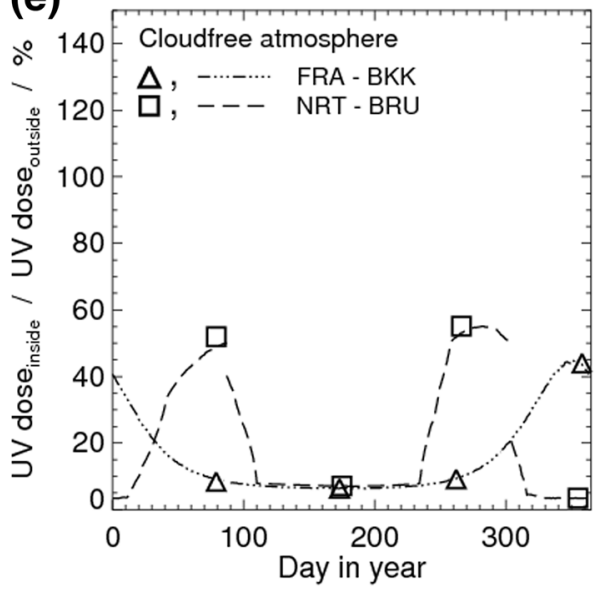

(b)

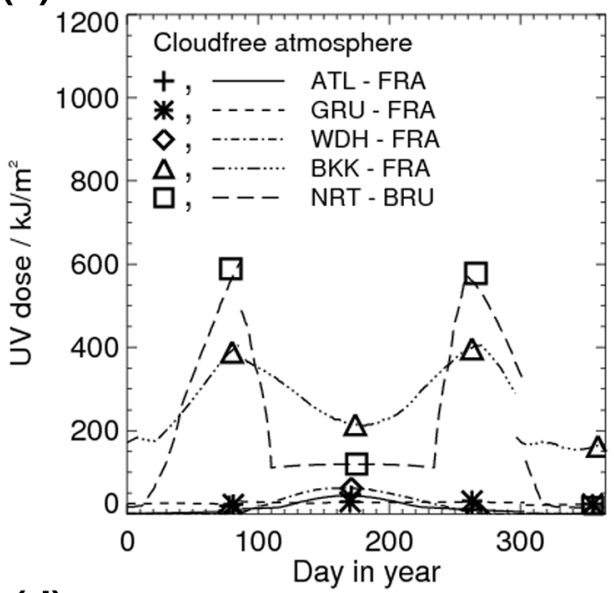

(d)

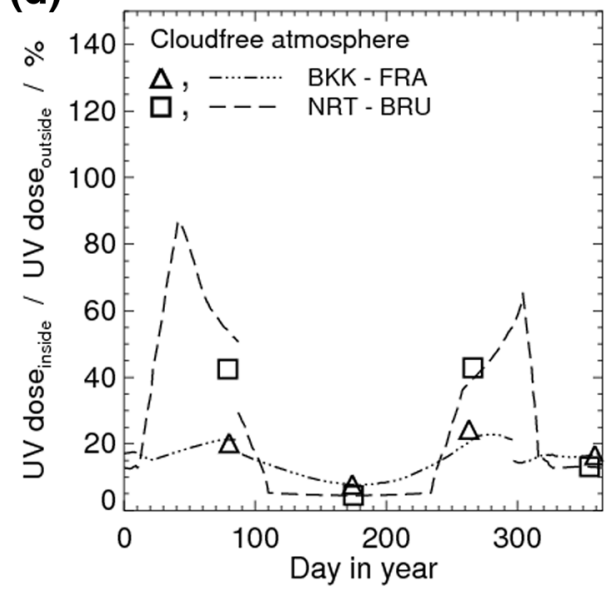

50-90\%) when compared to doses outside the airplane on the same day and calculated for the same time interval. One main limiting factor is the windscreen geometry cutting-off solar zenith angles less than $30^{\circ}$. Note again, simulations are performed for the spectral range $290-400 \mathrm{~nm}$, but UV doses inside the cockpit are de facto UV-A doses since the windscreen blocks UV-B radiation. Detrimental effects of $\mathrm{UV}-\mathrm{B}$ radiation are not at all considered here.

\section{Summary and concluding remarks}

This study addresses the question how much of the ambient UV radiation is transmitted into the cockpit of commercial jets during flight. Based on radiative transfer calculations UV irradiances inside and outside the cockpit are calculated by taking into account the optical properties and approximated windscreen geometry as well as relevant 
parameters of the atmospheric-surface system like cloudiness and snow cover. By the use of MOZAIC geolocalization data of selected intercontinental flights, cumulative UV doses inside and outside the cockpit are estimated and compared. Results indicate the dependencies of UV doses on flight route, flight time, and the day of the year. The main items of this study may be summarized as follows:

- The intensity of UV radiation inside a cockpit strongly depends on whether the direct sun is entering the cockpit or not.

- In case only diffuse UV radiation reaches the interior of the cockpit irradiances amount to about 5\% the irradiances outside the aircraft.

- In case diffuse and direct UV radiation reaches the interior of the cockpit UV irradiances amount to $50-100 \%$ the irradiances outside. Percentages increase with increasing solar zenith angle, but absolute irradiances decrease with increasing solar zenith angle.

- A water cloud in the lower troposphere below an aircraft flying at $10 \mathrm{~km}$ altitude increases UV irradiances inside the cockpit by about $7 \%$ when compared to the cloud free atmosphere. A snow covered surface causes a similar increase at maximum.

- Flight route parameters and the day of the year determine the solar position relative to the aircraft heading and in combination with atmospheric conditions they determine the altitude dependent contribution of UV irradiances to UV doses integrated from takeoff to landing time.

- For flight routes selected in this study UV doses inside the cockpit and under conditions of a cloud free atmosphere amount to maximum $60 \%$ the doses outside at the same flight altitude. In most cases, however, percentages lie between about 10 and $40 \%$.

- It is obvious that one has to distinguish between the UV radiation inside and outside an aircraft cockpit when assessing potential UV exposures of airliner pilots. Although ambient UV radiation is significantly higher at flight altitude and may be further enhanced by reflecting snow surfaces or cloud layers only a fraction of it will be transferred into the interior of a cockpit.

Simulation results are certainly afflicted with uncertainties. For example, the windscreen geometry is simplified compared to real jet windscreens which show a more complex design and differ depending on aircraft type. Although the assumed geometry is suitable to describe the order of magnitude of UV radiation reaching the cockpit, some quantitative numbers may point to uncertainties introduced by simplification and variability of windscreens. Considered is a case leading to higher UV exposure.
Opening the viewing azimuth angle of the windscreen from $\Delta \varphi=150^{\circ}$ to $\Delta \varphi=180^{\circ}$ and the viewing zenith angle from $\Delta \delta=50^{\circ}$ to $\Delta \delta=55^{\circ}$ (Fig. 2) would enlarge the entire solid angle by about $14 \%$. In any case this results in a higher contribution of diffuse radiation to UV exposure, but it also expanses the angular sector for direct radiation. The windscreen enlargement enhances the diffuse radiation by less than $5 \%$. It should be kept in mind that inside a cockpit the diffuse component contributes by about only $15 \%$ to the total of direct and diffuse radiation. During the flights NRT-BRU in March and September diffuse as well as direct radiation is entering the cockpit. For these flights UV doses would increase by about $9 \%$ on 30.03 .1998 and by about $14 \%$ on 24.09 .1998 .

Modern airline aircraft types are fitted with visors for the front windshields and roller binds for the side windows. These are in common-place use in flight and are likely to be used to block direct solar radiation. Such devices imply the potential of reducing UV exposures during flight significantly. For example, blocking out the direct component of solar UV radiation means that at $10 \mathrm{~km}(0 \mathrm{~km})$ altitude and for sza $=66^{\circ} \mathrm{UV}$ irradiances inside the cockpit are reduced by about $86 \%(80 \%)$. A subtraction of the direct radiation during the entire flight NRT-BRU would reduce the UV dose from 657 to $99 \mathrm{kJm}^{-2}$ on 30.03 .1998 and from 573 to $91 \mathrm{kJm}^{-2}$ on 24.09.1998. For the flight FRAATL on 22.12.1999, UV doses would correspondingly decrease from 291 to $51 \mathrm{kJm}^{-2}$. It should be mentioned that sunglasses, certain types of spectacles and contact lenses are also suitable to reduce ocular UV-A exposure, but ocular protection was not within the scope of this study.

Under real flight conditions, especially during the takeoff and landing phases, aircrafts are changing angles of pitch and roll. This has not been considered and any estimation of exposure during these phases of flight is likely to be less accurate. On the other hand and as far as long-range flights of about $10 \mathrm{~h}$ flying time are considered irradiances during ascent and descent phases amount with a temporal share of about $10 \%$ to the entire UV dose.

The target area receiving incident radiation is assumed to be fixed in position and vertical orientation. Usually the skin elements of a pilot are in movement which implies temporal variations of the effective solid angle spanned by the windscreen and the target receiving incident radiation. Indeed, time dependent effects of pitch and role as well as skin orientation have in common that they affect the preconditions whether direct solar UV radiation impacts the pilot's skin or not. However, integrated over flying time they might average out to a certain extent. Actually the radiative interaction between the pilot's body and the UV radiation field during flight defines a rather complex 3D 
(4D) problem and might be approached by extensive UV measurements, possibly accompanied by $3 \mathrm{D}$ numerical radiative transfer simulations. But this was not the objective of this study.

UV doses for the overseas flights are calculated for a cloud free atmosphere. Considering the real state of the atmospheric-surface system at the time of flight would mean to acquire all relevant input data in adequate temporal and spatial resolution. This is expensive and has not been realized. However, irradiance profiles shown in Fig. 3 may give a hint how calculated UV doses deviate due to the effects of clouds or snow and ice on the ground.

As mentioned, wavelength integrated UV irradiances behind the windscreens consist of spectral contributions from the UV-A part of the spectrum, whereas outside the cockpit ambient radiation also includes the UV-B radiation. Comparing both implies, in the first instance, a disregard of the shorter UV-B wavelengths where photons are carrying higher energies especially associated with adverse effects on human skin or eye. On the other hand, UV-B irradiances amount to only about $1 \%$ the irradiances integrated over the total range from 280 to $400 \mathrm{~nm}$. A comparative examination of ambient and cockpit UV irradiances as done in this study is therefore particularly appropriate for drawing conclusions concerning the UV-A radiation. Nevertheless, presented results should be interpreted in view of the fact that only three different windscreen transmittances of commercial jets have been considered with the effect of one being studied in more detail. More spectral windscreen measurements might contribute to a more precise and representative assessment of the uncertainties of UV exposures inside airliner cockpits.

UV action spectra accounting for biologically damaging effects within the UV-A have not been taken into account and cumulative individual UV doses pilots absorbed during their occupational life have not been quantified. Such doses depend on the entire time a pilot spent in a cockpit, on the number of flights along specific routes, and on the specific windscreen properties of the aircraft types he has operated. Finally, the individual skin type of a pilot is certainly of relevance and its inclusion might be subject of a further investigation.

Acknowledgements The author acknowledges the strong support of the European Commission, Airbus, and the Airlines (Lufthansa, AirFrance, Austrian, Air Namibia, Cathay Pacific, Iberia and China Airlines so far) who carry the MOZAIC or IAGOS equipment and perform the maintenance since 1994. MOZAIC is presently funded by INSU-CNRS (France), Météo-France, Université Paul Sabatier (Toulouse, France) and Research Center Jülich (FZJ, Jülich, Germany). IAGOS has been and is additionally funded by the EU projects IAGOS-DS and IAGOS-ERI. The MOZAIC-IAGOS database is supported by ETHER (CNES and INSU-CNRS). Data are also available via Ether web site http://www.pole-ether.fr. Furthermore, the author would like to thank Klaus Gierens (DLR) for fruitful discussions and his constructive comments.

\section{References}

1. Kimlin, M.G., Tenkate, T.D.: Occupational exposure to ultraviolet radiation: the duality dilemma. Rev. Environ. Health. 22(1), 1-38 (2007)

2. WHO, World Health Organization, (C2015. http://www.who.int/ uv/health/en/ and related links and reports (2015). Accessed 15 June 2015

3. Diffey, B.L., Roscoe, A.H.: Exposure to solar ultraviolet radiation in flight. Aviat. Space Environ. Med. 61(11), 1032-1035 (1990)

4. Chorley, A.D., Evans, B.J.W., Benwell, M.J.: Civilian pilot exposure to ultraviolet and blue light and pilot use of sunglasses, review article. Aviat. Space Environ. Med. 82(9), 895-900 (2011). doi:10.3357/ASEM.3034

5. Huang, X.X., Bernerd, F., Halliday, G.M.: Ultraviolet A within sunlight induces mutations in the epidermal basal layer of engineered human skin. Am. J. Pathol. 174(4), 1534-1543 (2009). doi:10.2353/ajpath.2009.080318

6. Kappes, U.P., Luo, D., Potter, M., Schulmeister, K., Rünger, T.M.: Short- and long-wave UV light (UVB and UVA) induce similar mutations in human skin cells. J. Invest. Dermatol. 126, 667-675 (2006). doi:10.1038/sj.jid.5700093

7. Nakagawara, V.B., Montgomery, R.W., Marshall, J.W.: Optical radiation, transmittance of aircraft windscreens and pilot vision. Federal Aviation Administration, Washington, DC (2007)

8. Sanlorenzo, M., Mackenzie, R., Wehner, M.R., Linos, E., Kornak, J., Kainz, W., Posch, C., Vujic, I., Johnston, K., Gho, D., Monico, G., McGrath, J.T., Osella-Abate, S., Quaglino, P., Cleaver, J.E., Ortiz-Urda, S.: The risk of melanoma in airline pilots and cabin crew: a meta-analysis, published online September 3, 2014. JAMA Dermatol (2014). doi:10.1001/jama dermatol.2014.1077

9. Sanlorenzo, M., Vujic, I., Posch, C., Cleaver, J.E., Quaglino, P., Ortiz-Urda, S.: The risk of melanoma in pilots and cabin crew: UV measurements in flying airplanes. JAMA Dermatol. 151(4), 450-452 (2015). doi:10.1001/jamadermatol.2014.4643

10. Chorley, A., Higglet, M., Baczynska, K., Hunter, R., Khazova, M.: Measurements of pilots' occupational solar UV exposure. J. Photochem. Photobiol. 90(4), 935-940 (2014). doi:10.1111/ php. 12269

11. Liu, Q., Weng, F.: Advanced doubling adding method for radiative transfer in planetary atmospheres. J. Atmos. Sci. 63, 3459-3465 (2006). doi:10.1175/JAS3808.1

12. Meerkötter, R., Degünther, M.: A radiative transfer case study for 3-d cloud effects in the UV. Geophys. Res. Lett. 28(9), 1683-1686 (2001)

13. Meerkötter, R., Verdebout, J., Bugliaro, L., Edvardsen, K., Hansen, G.: An evaluation of cloud affected UV radiation from polar orbiting and geostationary satellites at high latitudes. Geophys. Res. Lett. 30(18), 1956 (2003). doi:10.1029/ 2003GL017850

14. Anderson, G.P., Clough, S.A., Kneizys, F.X., Chetwynd, J.H., Shettle, E.P.: AFGL atmospheric constituent profiles $(0-120 \mathrm{~km})$. No. AFGL-TR-860110, Air Force Geophysics Lab, Hanscom, AFB, MA (1986)

15. Meinander, O., Kazadzis, S., Arola, A., Riihelä, A., Räisänen, P., Kivi, R., Kontu, A., Kouznetsov, R., Sofiev, M., Svensson, J., Suokanerva, H., Aaltonen, V., Manninen, T., Roujean, J.-L., Hautecoe, O.: Spectral albedo of seasonal snow during intensive melt period at Sodankylä, beyond the Arctic Circle. Atmos. 
Chem. Phys. 13, 3793-3810 (2013). http://www.atmos-chemphys.net/13/3793/2013/. doi:10.5194/acp-13-3793-2013

16. Kinne, S., O'Donnel, D., Stier, P., Kloster, S., Zhang, K., Schmidt, H., Rast, S., Giorgetta, M., Eck, T.F., Stevens, B.: MAC-v1: a new global aerosol climatology for climate studies. JAMES. 5, 704-740 (2013). doi:10.1002/jame.20035
17. Report, AIRBUS, A319/A320/A321 Flight deck and systems briefing for pilots, STL945.7136/97, issue September (1998)

18. Report, AIRBUS, A340 Flight deck and systems briefing for pilots, STL472.502/90, issue 6 (2000) 